\title{
Comparison of effects of anti-angiogenic agents in the zebrafish efficacy-toxicity model for translational anti-angiogenic drug discovery
}

This article was published in the following Dove Press journal:

Drug Design, Development and Therapy

19 August 2014

Number of times this article has been viewed

\author{
Geetanjali Chimote' \\ Jayasree Sreenivasan' \\ Nilambari Pawar' \\ Jyothi Subramanian² \\ Hariharan \\ Sivaramakrishnan ${ }^{3}$ \\ Somesh Sharma ${ }^{1,3}$ \\ 'Department of Pharmacology, \\ ${ }^{2}$ Department of Modeling and \\ Simulation, ${ }^{3}$ Department of \\ Medicinal Chemistry, Piramal Life \\ Sciences Limited, Mumbai, India
}

Background: Anti-angiogenic therapy in certain cancers has been associated with improved control of tumor growth and metastasis. Development of anti-angiogenic agents has, however, been saddled with higher attrition rate due to suboptimal efficacy, narrow therapeutic windows, or development of organ-specific toxicities. The aim of this study was to evaluate the translational ability of the zebrafish efficacy-toxicity model to stratify anti-angiogenic agents based on efficacy, therapeutic windows, and off-target effects to streamline the compound selection process in anti-angiogenic discovery.

Methods: The embryonic model of zebrafish was employed for studying angiogenesis and toxicity. The zebrafish were treated with anti-angiogenic compounds to evaluate their effects on angiogenesis and zebrafish-toxicity parameters. Angiogenesis was measured by scoring the development of subintestinal vessels. Toxicity was evaluated by calculating the median lethal concentration, the lowest observed effect concentration, and gross morphological changes. Results of efficacy and toxicity were used to predict the therapeutic window.

Results: In alignment with the clinical outcomes, the zebrafish assays demonstrated that vascular endothelial growth factor receptor (VEGFR) inhibitors are the most potent anti-angiogenic agents, followed by multikinase inhibitors and inhibitors of endothelial cell proliferation. The toxicity assays reported cardiac phenotype in zebrafish treated with VEGFR inhibitors and multikinase inhibitors with VEGFR activity suggestive of cardiotoxic potential of these compounds. Several other pathological features were reported for multikinase inhibitors suggestive of off-target effects. The predicted therapeutic window was translational with the clinical trial outcomes of the anti-angiogenic agents. The zebrafish efficacy-toxicity approach could stratify anti-angiogenic agents based on the mechanism of action and delineate chemical structure-driven biological activity of anti-angiogenic compounds.

Conclusion: The zebrafish efficacy-toxicity approach can be used as a predictive model for translational anti-angiogenic drug discovery to streamline compound selection, resulting in safer and efficacious anti-angiogenic agents entering the clinics.

Keywords: angiogenesis, therapeutic window, VEGFR inhibitors, zebrafish toxicity assay

\section{Introduction}

Tumor angiogenesis has been the focus area in cancer drug discovery for over a decade. ${ }^{1}$ Phenotypic and genetic differences between tumor and nontumor endothelial cells represented a rational strategy for developing anti-angiogenic agents as targeted drugs with limited systemic side effects. ${ }^{2}$ The major classes of anti-angiogenic agents in clinical use and evaluation include vascular endothelial growth factor receptor (VEGFR) inhibitors like sunitinib, SU5416, vatalanib, vandetanib, tivozanib, pazopanib, motesanib, cabozantinib, and axitinib; followed by multikinase inhibitors
Correspondence: Geetanjali Chimote Department of Pharmacology, Piramal Life Sciences Limited, I Nirlon Complex, Off Western Express Highway, Goregaon East, Mumbai 400063, Maharashtra, India Tel +912230818112

$\mathrm{Fax}+912230818036$

Email geetanjali.chimote@piramal.com 
like sorafenib, regorafenib, flavopiridol, and suramin; and inhibitors of endothelial cell proliferation like combretastatin, TNP-470, and thalidomide (Table 1). ${ }^{3}$ Anti-angiogenic activity is also evaluated for endothelial growth factor receptor tyrosine-kinase inhibitors like erlotinib, which is known to inhibit angiogenesis by functional cross-talk with the vascular endothelial growth factor (VEGF) pathway.

Contrary to expectations, initial anti-angiogenic drug discovery efforts met with high attrition rates in the clinics, attributed to narrow therapeutic windows, off-target effects, and lack of desired efficacy. ${ }^{4-6}$ The existing angiogenesis models used to study angiogenesis could not address the aforementioned areas of concern during compound screening in early drug discovery. The existing lacunae in antiangiogenic drug discovery necessitates introduction of an efficacy-toxicity model to characterize effectiveness of anti-angiogenic agents and establish therapeutic windows and off-target potential during the anti-angiogenic compound screening process. Therefore, the present study was undertaken to evaluate whether embryonic zebrafish will provide an alternative preclinical efficacy-toxicity model for translational anti-angiogenic drug discovery.

Angiogenesis is evaluated by several phenotypic screens in drug discovery, such as in vitro cell-based and in vivo whole-organism approaches. The utility of in vitro angiogenic assays like the endothelial cell migration and tube formation as translational models is limited by their inability to simulate the complexity of the in vivo milieu and demonstrate differential behavior of the heterogeneous endothelial cells. ${ }^{7}$ The in vivo animal models like the matrigel plug assay, the chick chorioallantoic membrane (CAM) assay, or the corneal angiogenesis assay maintain biological complexity, but are low throughput and semi-quantitative, requiring considerable amount of drug and personnel engagement to be utilized extensively for anti-angiogenic compound screening. ${ }^{8}$

The zebrafish, small freshwater fish, exhibit highly characteristic blood-vessel patterning and a short period of development of blood vessels (ie, 96 hours post-fertilization [hpf]). Vasculogenesis in the zebrafish is initiated as early as $12 \mathrm{hpf}$, and by $24 \mathrm{hpf}$ a simple circulatory loop consisting of major vessel-like dorsal aorta and axial vein is established. By $24 \mathrm{hpf}$, development of angiogenic sprouts like the subintestinal vessels (SIVs) is initiated to establish angiogenesis in the developing gut, allowing evaluation of anti-angiogenic agents. ${ }^{9}$ Further, zebrafish as a model organism exhibit genetic and functional conservation across angiogenic pathways. The major modulators of angiogenesis,

Table I Anti-angiogenic agents with different mechanisms of action

\begin{tabular}{|c|c|c|c|}
\hline Anti-angiogenic agent & Mechanism of action & $\begin{array}{l}\text { Current clinical } \\
\text { status }\end{array}$ & Associated clinical toxicities \\
\hline \multicolumn{4}{|l|}{ VEGF/VEGFR inhibitors } \\
\hline Bevacizumab & Recombinant monoclonal antibody against human VEGF & Approved & Delayed wound healing, bleeding \\
\hline Sunitinib & VEGFR inhibitor & Approved & Cardiotoxicity \\
\hline Motesanib & VEGFR, PDGFR, and SCF receptor inhibitor & In clinical trials & Cardiotoxicity \\
\hline Pazopanib & VEGFR-I, $-2,-3$, PDGFR- $\alpha / \beta$ inhibitor & Approved & Cardiotoxicity \\
\hline Tivozanib & VEGFR inhibitor & In clinical trials & Cardiotoxicity \\
\hline Vandetanib & VEGFR, EGFR inhibitor & Approved & Cardiotoxicity \\
\hline Vatalanib & VEGFR, PDGFR, and c-KIT receptor inhibitor & Withdrawn & Multiple systemic toxicities \\
\hline SU54I6 & VEGFR-2 inhibitor & Withdrawn & Multiple systemic toxicities \\
\hline Axitinib & VEGFR I-3 receptor inhibitor, c-KIT, and PDGFR inhibitor & Approved & Cardiotoxicity \\
\hline Cabozantinib & VEGFR-2 inhibitor & Approved & Multiple systemic toxicities \\
\hline \multicolumn{4}{|l|}{ Multikinase inhibitors } \\
\hline Sorafenib & Multikinase inhibitor & Approved & Cardiotoxicity \\
\hline Flavopiridol & $\begin{array}{l}\text { Inhibition of cyclin-dependent kinases with anti-angiogenic } \\
\text { activity }\end{array}$ & Withdrawn & Multiple systemic toxicities \\
\hline Suramin & Inhibition of bFGF & Withdrawn & Multiple systemic toxicities \\
\hline Regorafenib & Multikinase inhibitor & Approved & Cardiotoxicity and hepatotoxicity \\
\hline \multicolumn{4}{|c|}{ Inhibitors of endothelial cell proliferation } \\
\hline Thalidomide & $\begin{array}{l}\text { Endothelial cell apoptosis, inhibition of bFGF-induced } \\
\text { angiogenesis }\end{array}$ & Approved & Teratogenic \\
\hline TNP-470 & Inhibition of endothelial cell proliferation and migration & Withdrawn & Neurotoxicity \\
\hline Combretastatin & Vascular disrupting agent, endothelial cell apoptosis & Withdrawn & Multiple systemic toxicities \\
\hline Erlotinib & Tyrosine-kinase inhibitor of EGFR & Approved & Multiple systemic toxicities \\
\hline
\end{tabular}

Abbreviations: VEGF, vascular endothelial growth factor; VEGFR, vascular endothelial growth factor receptor; PDGFR, platelet-derived growth factor receptor; SCF, stem cell factor; bFGF, basic fibroblast growth factor; EGFR, endothelial growth factor receptor. 
tyrosine-kinase domains of vascular endothelial growth factor receptor 2 (VEGFR2) and VEGF, are conserved. ${ }^{10,11}$

Chemical genetic approaches utilizing chemical inhibitors for each functional target have been studied for antiangiogenic compounds in the zebrafish; however, studies have been limited to a few specific compounds. ${ }^{12}$ There is thus a need to characterize the translational effects of different classes of anti-angiogenic agents in the zebrafish to typify a mechanism-based class effect on anti-angiogenic efficacy and toxicity, facilitate establishment of therapeutic windows, and identify off-target effects, which are key reasons impeding drug development at a later stage. ${ }^{13}$ To the best of our knowledge this is the first attempt to achieve the aforesaid.

To explore how the zebrafish angiogenesis and toxicity assays complement each other we analyzed the effects of a range of angiogenesis inhibitors with different mechanisms of action (VEGFR inhibitors, multikinase inhibitors, inhibitors of endothelial cell proliferation) on the zebrafish embryos. A mechanism-based class-specific anti-angiogenic efficacy and toxicity was observed in the zebrafish embryos, with VEGFR inhibitors being the most potent inhibitors of angiogenesis, followed by multikinase inhibitors and inhibitors of endothelial cell proliferation. The zebrafish toxicity assays were also able to delineate mechanism-based differences in the toxicity profile. VEGFR inhibitors expressed progressive pericardial edema, while treatment with multikinase inhibitors was associated with development of several toxicities and gross morphological changes, akin to their clinical toxicities. Anti-angiogenic agents exhibiting acceptable therapeutic windows in the clinics exhibited therapeutic windows in the zebrafish efficacy-toxicity model. Anti-angiogenic agents like TNP-470 and SU5416, associated with dose-limiting toxicities in clinical trials, also demonstrated lack of therapeutic windows in the zebrafish.

These results suggest that complementary use of zebrafish efficacy-toxicity assays could be a promising strategy in translational drug discovery to streamline compound selection process, reduce costs, and improve clinical success of anti-angiogenic drug discovery.

\section{Materials and methods Materials}

The anti-angiogenic compounds SU5416, combretastatin, thalidomide, TNP-470, flavopiridol, suramin, paclitaxel, and sorafenib were purchased from Sigma-Aldrich Co. (St Louis, MO, USA); vatalanib, motesanib, pazopanib, tivozanib, and vandetanib were purchased from LC Laboratories (Woburn, MA, USA). Axitinib, cabozantinib, and regorafenib were purchased from Selleck Chemicals (Houston, TX, USA). All other chemicals used in this study were obtained from Sigma-Aldrich Co., unless mentioned otherwise.

\section{Zebrafish}

Permission to work on zebrafish was obtained from the Institutional Animal Ethics Committee, India, under registration No (29/1999/CPCSEA). Wild-type zebrafish were maintained in a temperature-controlled room at $28^{\circ} \mathrm{C}$ with a 14:10 hour day/night cycle. The zebrafish were randomly selected for mating and housed in breeding tanks, in the male-to-female ratio of $2: 1$. The embryos were collected from the breeding trap and washed thoroughly to eliminate any debris lying at the bottom of the tank. The zebrafish embryos obtained post-spawning were staged, ${ }^{14}$ dispensed in distilled water, and maintained in an incubator at $28.5^{\circ} \mathrm{C} \pm 0.5^{\circ} \mathrm{C}$ in the dark.

\section{Zebrafish subintestinal vessel angiogenesis assay}

The zebrafish embryos were maintained in distilled water in 12- well cell culture plates and each well contained 20 embryos. At $24 \mathrm{hpf}$ the embryos were dechorionated with pronase treatment and immersed in a solution containing $0.003 \%$ phenyl-1-thio urea in distilled water to prevent pigmentation. Each treatment group contained 20 embryos per test concentration. A negative control group containing zebrafish embryos in distilled water and a vehicle treatment group that was treated with $0.1 \%$ dimethyl sulfoxide (DMSO) was used for each test compound.

The ability of anti-angiogenic agents to inhibit subintestinal vessel development was evaluated. The endothelial cells of the SIVs were stained for alkaline phosphatase at $96 \mathrm{hpf}$, and with fluorescein lectin (Alexa Fluor ${ }^{\circledR} 488$ conjugate; Thermo Fisher Scientific, Waltham, MA, USA) at $72 \mathrm{hpf}$ as described elsewhere. ${ }^{9,13,15}$ SIV angiogenesis inhibition was scored on the basis of vessel length extension from the somite to the angiogenic basket, number of interconnecting angiogenic vessels within the basket, and aberrant angiogenic projections at the end of $96 \mathrm{hpf}$ (Figure 1). The aforementioned parameters were the most sensitive and frequently altered parameters in the zebrafish angiogenesis assays and hence were included for stratifying the anti-angiogenic effects. The effective anti-angiogenic concentration $\left(\mathrm{EC}_{50}\right)$ was defined as the compound concentration that led to a $50 \%$ reduction in the vessel length as compared with the mean vessel length for the controls. In addition to evaluating SIV angiogenesis, the developing zebrafish embryo permit assessment of effect 


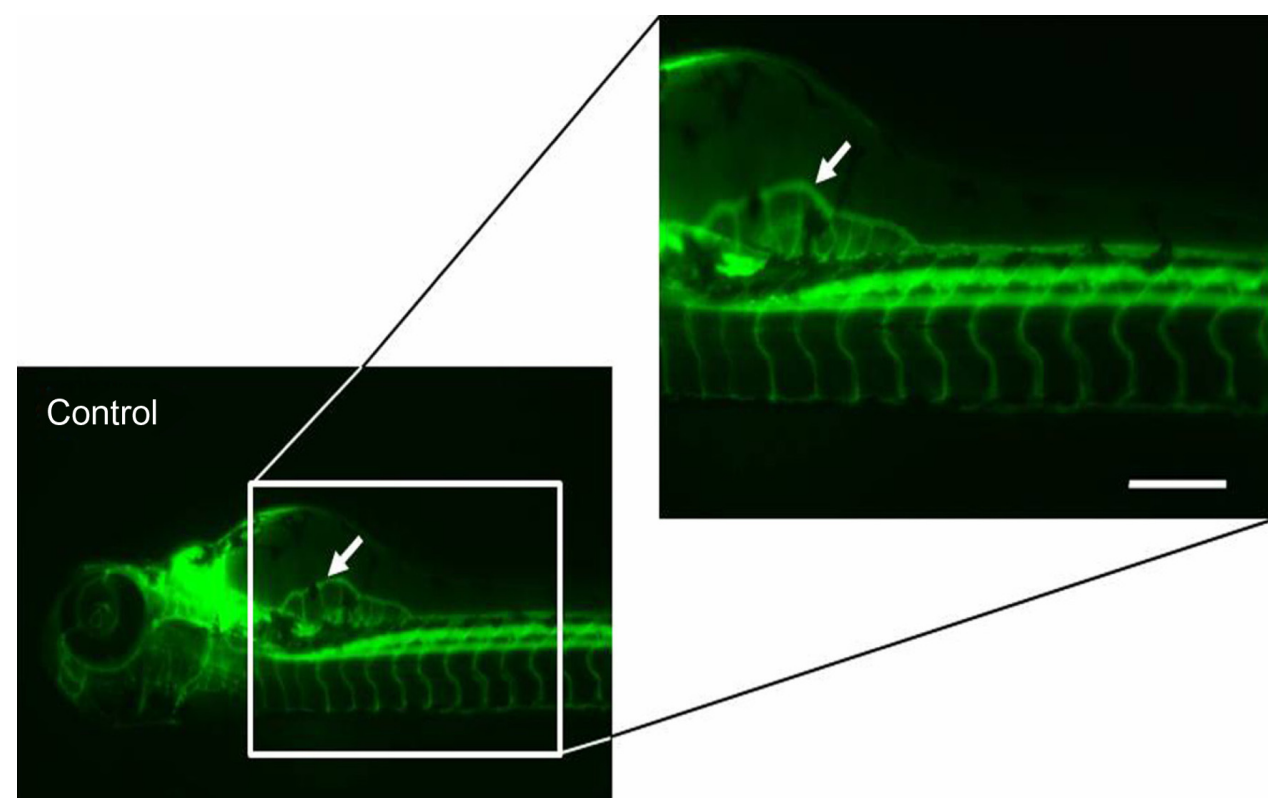

Figure I Scoring parameters: subintestinal vessel angiogenesis.

Notes: Number of vessels within the basket numerically counted and length of the angiogenic basket drug represented by the arrow. Vessels stained with lectin injections (Alexa Fluor $^{\circledR} 488$ conjugate; Molecular Probes, Eugene, OR, USA): $2 \mathrm{~nL}$ of $5 \mathrm{mg} / \mathrm{mL}$ in developing embryonic pumping heart at 72 hours post-fertilization. Scale bar denotes $20 \mu \mathrm{m}$.

of anti-angiogenic agents on the ongoing vasculogenesis of the circulatory loop consisting of dorsal aorta and axial vein, which is established by $24 \mathrm{hpf}$.

\section{Zebrafish toxicity assay}

The zebrafish embryos were collected at $24 \mathrm{hpf}$ and randomly divided into naïve control (embryos maintained in distilled water), vehicle control (embryos treated with $0.1 \%$ DMSO), and treatment groups. Each group had 20 embryos per test concentration. Stock solutions of all the anti-angiogenic compounds were prepared in $0.1 \%$ DMSO as a solubilizing agent. The $\mathrm{pH}$ of the final test solutions was maintained between 6.8 and 8 . The test solutions were bath-applied to the zebrafish embryos and renewed every 24 hours. ${ }^{16}$ The zebrafish embryos were maintained in an incubator at $28^{\circ} \mathrm{C}$ and were read at 48 and $96 \mathrm{hpf}$ for organ-specific toxicity and other toxicity parameters. Each of the anti-angiogenic compounds was evaluated at five different concentrations, taking into account the compound solubility limits in the test medium (distilled water). Each compound was evaluated in three independent biological experiments.

\section{Toxicity parameters}

The toxicity was evaluated by calculating the median lethal concentration $\left(\mathrm{LC}_{50}\right)$, which was the treatment concentration that resulted in 50\% mortality of the embryos over a period of 96 hours. The no observed effect concentration (NOEC) and the lowest observed effect concentration (LOEC) were experimentally derived. The NOEC was an experimentally derived concentration that had no untoward effects on the zebrafish larval morphology, whereas the LOEC was the treatment concentration that resulted in pathological effects on $50 \%$ of the zebrafish larval population. The NOEC, therefore, represents safe drug concentrations in the zebrafish while the LOEC represents sub-lethal concentrations having adverse effects in the zebrafish.

\section{Therapeutic window}

The therapeutic window was considered as the concentration range between effective anti-angiogenic concentrations $\left(\mathrm{EC}_{50}\right)$ and lowest concentrations of anti-angiogenic agents resulting in pathological effects (LOEC). The therapeutic window was plotted as the range between $\mathrm{LOEC}$ and $\mathrm{EC}_{50}$ of the anti-angiogenic agents. The therapeutic window defines the sub-lethal effects of the compounds with respect to its efficacious concentrations. The therapeutic window was evaluated to understand the sensitivity and specificity of zebrafish as an efficacy-toxicity model to predict the clinical outcomes. Since some of the antiangiogenic compounds have been dropped in clinical trials due to sub-lethal toxicities, the therapeutic window was calculated with respect to the LOEC concentrations.

\section{Statistical analysis}

The median lethal concentration $\left(\mathrm{LC}_{50}\right)$ was calculated from the total mortality in all three independent experiments and expressed as a percentage, in a sigmoidal 
concentration-response curve. The anti-angiogenic efficacy for each compound $\left(\mathrm{EC}_{50}\right)$ was determined from its concentration-response model (GraphPad Prism, version 5.03; GraphPad Software, Inc., San Diego, CA, USA). The therapeutic window for each compound was the range between the $\mathrm{EC}_{50}$ and the LOEC. The performance of the therapeutic window in the zebrafish was measured by the binary classification of sensitivity and specificity with respect to clinical trial outcome of the individual compounds.

\section{Results}

\section{Anti-angiogenic activity}

Many of the known angiogenesis inhibitors were active in the developing zebrafish at $96 \mathrm{hpf}$ (ie, 15/18). Figures $2 \mathrm{~A}$ and 3

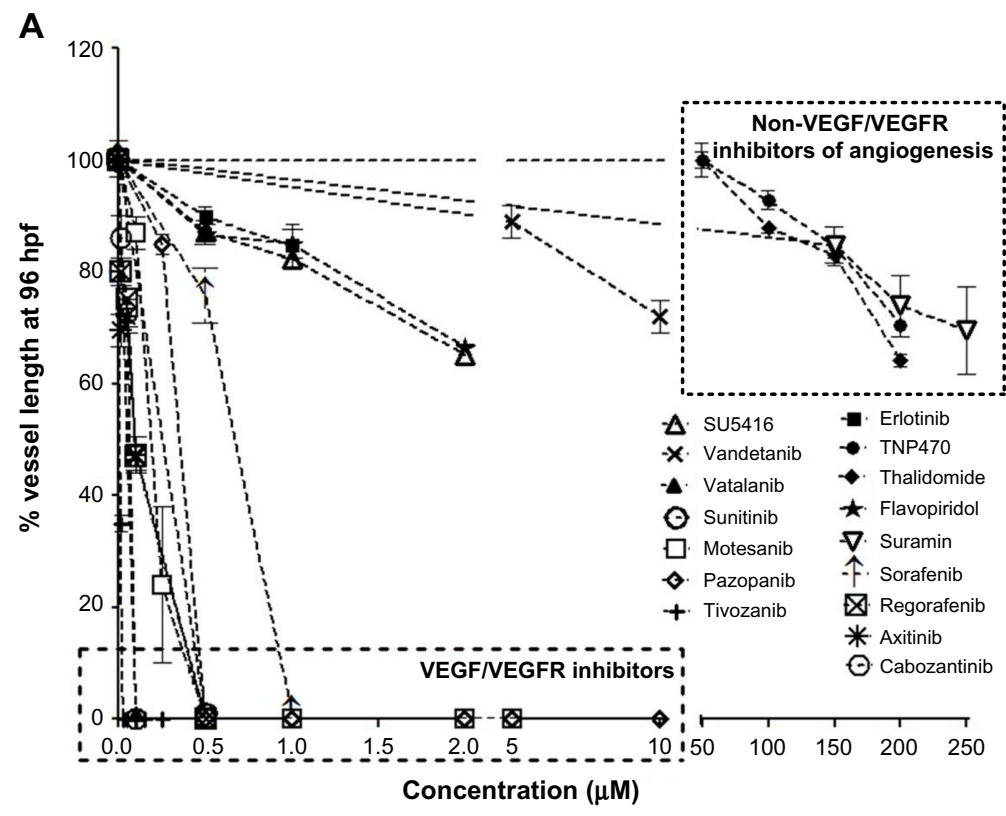

B

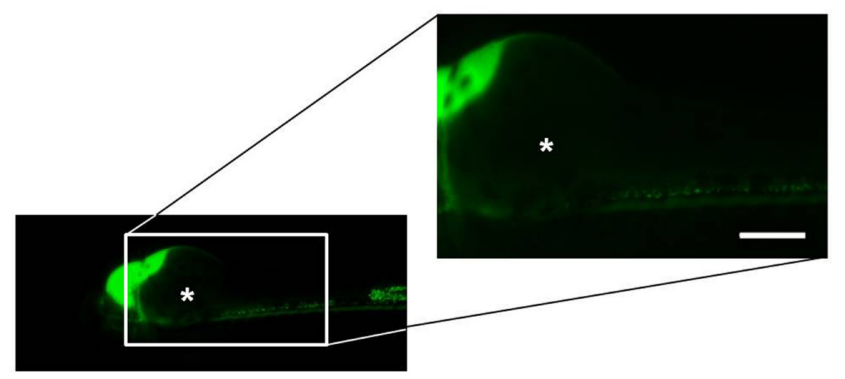

C

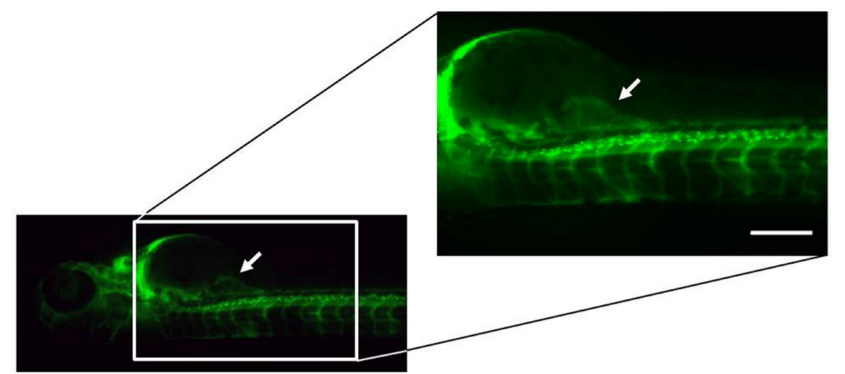

Figure 2 Anti-angiogenic effects after treatment with anti-angiogenic agents.

Notes: (A) Complete regression of SIVs upon treatment with VEGFR inhibitors (each compound was evaluated at NOEC or LOEC concentrations in three independent experiments with 20 embryos/concentration and continuous compound exposure for 72 hours). All data points are represented as mean \pm SEM. (B) Effect on SIV length and basket after treatment with anti-angiogenic agent (sorafenib, I $\mu \mathrm{M}$ ). Complete absence of SIVs $\left(^{*}\right)$ along with loss of intersegmental vessels. (C) Effect on SIV length and basket upon treatment with flavopiridol $(2 \mu \mathrm{M})$. Partial regression of SIVs (arrow). Vessels stained with lectin injections (Alexa Fluor ${ }^{\circledR} 488$ conjugate; Molecular Probes, Eugene, OR, USA): $2 \mathrm{~nL}$ of $5 \mathrm{mg} / \mathrm{mL}$ in developing embryonic pumping heart at $72 \mathrm{hpf}$. Scale bar denotes $20 \mu \mathrm{m}$.

Abbreviations: SIV, subintestinal vessel; VEGF, vascular endothelial growth factor; VEGFR, vascular endothelial growth factor receptor; NOEC, no observed effect concentration; LOEC, lowest observed effect concentration; SEM, standard error of the mean; hpf, hours post-fertilization. 


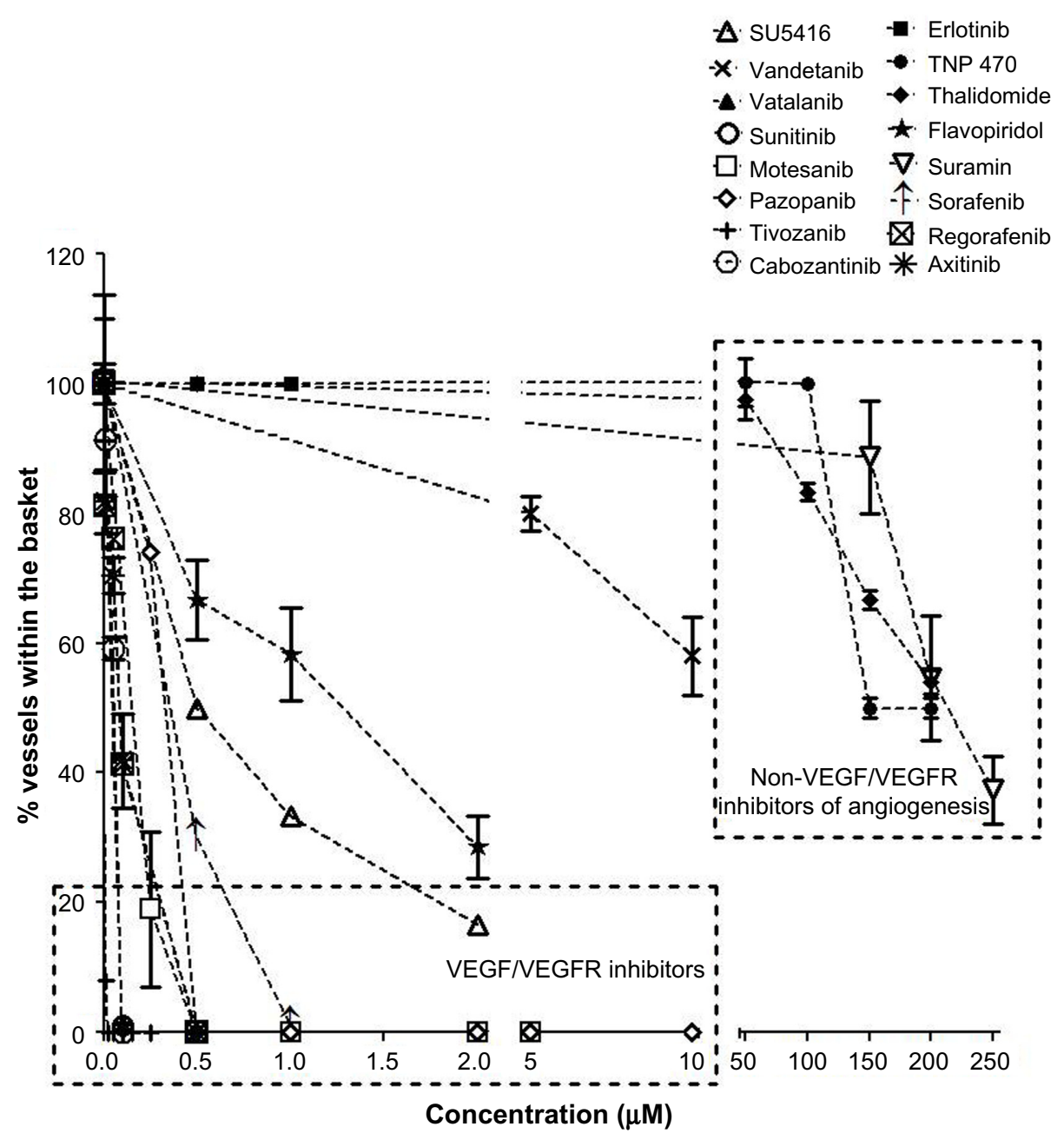

Figure 3 The number of vessels within the angiogenic basket posttreatment with anti-angiogenic agents.

Notes: Each compound was evaluated at NOEC or LOEC concentrations in three independent experiments with 20 embryos/concentration and continuous compound exposure for 72 hours. All data points are represented as mean \pm SEM.

Abbreviations: VEGF, vascular endothelial growth factor; VEGFR, vascular endothelial growth factor receptor; NOEC, no observed effect concentration; LOEC, lowest observed effect concentration; SEM, standard error of the mean.

illustrate the inhibitory effects of all the anti-angiogenic compounds on SIV formation in the zebrafish embryos at 96 hpf. Mechanism-based assortment of anti-angiogenic activity was observed between VEGFR inhibitors, followed by multikinase inhibitors and inhibitors of endothelial cell proliferation.

The VEGFR inhibitors were the most potent antiangiogenic agents. Treatment with all the VEGFR inhibitors resulted in complete regression of SIVs at concentrations $<1 \mu \mathrm{M}$ (Figure 2A). Tivozanib was the most potent VEGFR inhibitor and was associated with complete regression in SIVs at $5 \mathrm{nM}$, followed by cabozantinib, vatalanib, axitinib $\left(\mathrm{EC}_{50}=100 \mathrm{nM}\right)$, motesanib $\left(\mathrm{EC}_{50}=250 \mathrm{nM}\right)$, pazopanib, sunitinib $\left(\mathrm{EC}_{50}=500 \mathrm{nM}\right)$, and vandetanib $\left(\mathrm{EC}_{50}=10 \mu \mathrm{M}\right)$. Amongst the VEGFR inhibitors evaluated, incomplete SIV regression was observed only upon treatment with SU5416 and vandetanib (Figures 2A and 3). Treatment with SU5416 at $1 \mu \mathrm{M}$ was associated with $38 \%$ regression in SIV length and $85 \%$ decrease in the number of SIVs within the basket. Dose escalation beyond $2 \mu \mathrm{M}$ was limited due to dose-limiting toxicities.

Treatment with bevacizumab, a humanized monoclonal antibody to VEGF, resulted in extremely variable results. In the vehicle treated and untreated naïve controls, the SIVs developed as extensions forming a smooth basket-like structure at about $\sim 150-200 \mu \mathrm{m}$ from the somite with four to seven vessels within the angiogenic basket.

Amongst the multikinase inhibitors, sorafenib and regorafenib were the most potent inhibitors of angiogenesis (Figure 2A and B). Treatment with sorafenib and regorafenib 
resulted in complete regression of neoangiogenic SIV basket at $<1 \mu \mathrm{M}$. Treatment with flavopiridol resulted in $38 \%$ regression in SIV length and $72 \%$ decrease in the number of SIVs at $2 \mu \mathrm{M}$ (Figures 2A, C and 3). Treatment with suramin resulted in 30\% decrease in SIV length with $45 \%$ inhibition of vessels within the angiogenic basket. Dose escalation beyond $2 \mu \mathrm{M}$ for flavopiridol and beyond $200 \mu \mathrm{M}$ for suramin was not feasible due to dose-limiting toxicities. Concentrations above the NOEC were evaluated only for compounds with efficacious doses above the NOEC.

Amongst inhibitors of endothelial cell proliferation, treatment with TNP-470 as well as thalidomide caused limited regression of the SIVs (ie, about 20\%-30\%). Unlike other anti-angiogenic agents, treatment with TNP-470 was associated with formation of aberrant SIV extensions. Combretastatin treatment was associated with lack of antiangiogenic activity in the zebrafish embryos.

Erlotinib, an endothelial growth factor receptor tyrosinekinase inhibitor known to inhibit angiogenesis by functional cross-talk with the VEGF pathway, ${ }^{17}$ was evaluated in the zebrafish model for its anti-angiogenic potential. Treatment with erlotinib at $1 \mu \mathrm{M}$ inhibited the SIV length by $10 \%$ and had no effect on the number of SIVs within the arcade. Due to precipitation of erlotinib beyond this concentration, evaluation of anti-angiogenic activity at higher concentrations was not attempted.

All the anti-angiogenic agents demonstrated a dosedependent decrease in SIV angiogenesis.

\section{Toxicology profile and gross morphological effects}

Anti-angiogenic compounds with disparate anti-angiogenic mechanisms exhibited stratification of the lethal concentration ranges and dissimilar sub-lethal effects. Table 2 summarizes the zebrafish toxicology profile $\left(\mathrm{LC}_{50}, \mathrm{LOEC}\right.$, and NOEC concentrations) for anti-angiogenic compounds tested in this study.

For calculation of the aforementioned toxicology parameters, each compound was evaluated at five different

Table 2 Anti-angiogenic agents: profile in acute toxicity assay

\begin{tabular}{|c|c|c|c|c|}
\hline Anti-angiogenic agent & NOEC $(\mu \mathrm{M})$ & LOEC $(\mu \mathrm{M})$ & $\mathrm{LC}_{50}(\mu \mathrm{M})$ & Off-target effects \\
\hline \multicolumn{5}{|l|}{ VEGF/VEGFR inhibitors } \\
\hline Sunitinib & 0.5 & 1 & $1.4 \pm 0.12$ & Pericardial edema at I $\mu \mathrm{M}$ \\
\hline Motesanib & 0.1 & 0.5 & $1.4 \pm 0.0$ & Pericardial edema at $0.5 \mu \mathrm{M}$ \\
\hline Pazopanib & 0.5 & 1 & $3.2 \pm 0.0$ & Pericardial edema at I $\mu \mathrm{M}$ \\
\hline Tivozanib & 0.01 & 0.025 & $0.045 \pm 0.007 \mid$ & Pericardial edema at $0.025 \mu \mathrm{M}$ \\
\hline Vandetanib & 10 & 25 & $26.0 \pm 5.29$ & Pericardial edema at $25 \mu \mathrm{M}$ \\
\hline Vatalanib & 0.1 & 0.1 & $1.0 \pm 0.48$ & $\begin{array}{l}\text { Pericardial edema, yolk edema, jaw deformity } \\
\text { at } 0.1 \mu \mathrm{M} \text {, un-inflated swim bladder }\end{array}$ \\
\hline SU54I6 & 0.5 & 1 & $2.57 \pm 0.12$ & $\begin{array}{l}\text { Pericardial edema, yolk edema, blood } \\
\text { pooling, shortened body axis, jaw deformity } \\
\text { at I } \mu \mathrm{M} \text {, un-inflated swim bladder }\end{array}$ \\
\hline Axitinib & 0.1 & 0.5 & $1.0 \pm 0.0$ & Pericardial edema at $0.5 \mu \mathrm{M}$ \\
\hline Cabozantinib & 0.05 & 0.1 & $0.55 \pm 0.07$ & Pericardial edema at $0.1 \mu \mathrm{M}$ \\
\hline \multicolumn{5}{|l|}{ Multikinase inhibitors } \\
\hline Sorafenib & 0.2 & 1 & $2.0 \pm 0.09$ & Pericardial edema at I $\mu \mathrm{M}$ \\
\hline Flavopiridol & 0.5 & 1 & $2.32 \pm 0.05$ & $\begin{array}{l}\text { Yolk edema, blood pooling, shortened body } \\
\text { axis at } 2 \mu \mathrm{M} \text {, widespread tissue necrosis }\end{array}$ \\
\hline Suramin & 150 & 200 & $415.0 \pm 0.0$ & $\begin{array}{l}\text { Notochord deformities at } 200 \mu \mathrm{M} \text {, } \\
\text { pericardial edema }\end{array}$ \\
\hline Regorafenib & 0.05 & 0.1 & $0.625 \pm 0.04$ & Pericardial edema at $0.1 \mu \mathrm{M}$ \\
\hline \multicolumn{5}{|c|}{ Inhibitors of endothelial cell proliferation } \\
\hline Thalidomide & 200 & 250 & $342.0 \pm 10.91$ & $\begin{array}{l}\text { Abnormal fin development, decreased } \\
\text { otolith size }\end{array}$ \\
\hline TNP-470 & 50 & 100 & $205.0 \pm 1.71$ & Yolk edema at $\geq 100 \mu \mathrm{M}$ \\
\hline Combretastatin & 0.001 & 0.005 & $0.0059 \pm 0.0084$ & Coagulated eggs at $\geq 0.0 \mathrm{I} \mu \mathrm{M}$ \\
\hline Erlotinib & 1 & $\begin{array}{l}\text { Compound } \\
\text { precipitation }\end{array}$ & Not reached & None up to I $\mu \mathrm{M}$ \\
\hline
\end{tabular}

Notes: The toxicological parameters were derived from three independent biological replicates, wherein all the anti-angiogenic agents were evaluated at five different concentrations with a spacing factor of 2 , and 12 embryos/concentration in each biological replicate. Bevacizumab evaluation in the toxicological study led to results too inconclusive to derive any relevant toxicological parameters.

Abbreviations: VEGF, vascular endothelial growth factor; VEGFR, vascular endothelial growth factor receptor; NOEC, no observed effect concentration; LOEC, lowest compound concentration that had a significant effect on $50 \%$ of the zebrafish larval population when compared with the controls; $\mathrm{LC}_{50}$, median lethal concentration that resulted in $50 \%$ mortality of the embryos over a period of 96 hours. 
concentrations, with spacing factor of 2 . In each biological replicate, 12 embryos per concentration were used for each compound. All the experiments were conducted in triplicate to arrive at the $\mathrm{LC}_{50}$, LOEC, and NOEC concentrations. This was done to obtain statistically significant differentiation of morphological effects and toxicity parameters.

Treatment with VEGFR inhibitors exhibited $\mathrm{LC}_{50}$ at concentrations of $<2 \mu \mathrm{M}$ except treatment with vandetanib. Treatment with vandetanib resulted in an $\mathrm{LC}_{50}$ of $26 \mu \mathrm{M}$ at $96 \mathrm{hpf}$. Bevacizumab treatment was associated with too high a level of variability to derive a conclusive $\mathrm{LC}_{50}$ value.

Multikinase inhibitors exhibited $\mathrm{LC}_{50}$ at concentrations $<4 \mu \mathrm{M}$ except treatment with suramin. Treatment with suramin resulted in an $\mathrm{LC}_{50}$ of $415 \mu \mathrm{M}$ at $96 \mathrm{hpf}$. Though a multikinase inhibitor, the $\mathrm{LC}_{50}$ for suramin was about 200 -fold higher than the other multikinase inhibitors. This difference could be attributed to difference in the mechanism of angiogenesis inhibition and potential engagement of multiple targets resulting in toxic off-target effects. While flavopiridol and sorafenib inhibit angiogenesis by the VEGFR pathway, suramin potentiates its actions via basic fibroblast growth factor. Flavopiridol also is the most potent inhibitor of cyclindependent kinases involved in growth and development in comparison with suramin, which explains the different toxicity profiles. $\mathrm{LC}_{50}$ concentrations could not be achieved for erlotinib as it precipitated beyond $1 \mu \mathrm{M}$ concentration.

Amongst the endothelial cell proliferation inhibitors, treatment with thalidomide and TNP-470 exhibited $\mathrm{LC}_{50}$ at $342 \pm 10.91$ and $205 \pm 1.71 \mu \mathrm{M}$. Treatment with combretastatin exhibited the lowest $\mathrm{LC}_{50}$ amongst all the anti-angiogenic compounds at $5 \mathrm{nM}$.

The $\mathrm{LC}_{50}$ concentrations emphasized the toxicity differences between the VEGFR inhibitors, the multikinase inhibitors, and the endothelial cell proliferation inhibitors. We report to The results of this study demonstrate that the $\mathrm{LC}_{50}$ ranges were comparable for compounds with similar mechanisms of action.

Angiogenesis inhibitors elicited different gross morphological responses in their toxicity profile depending upon the biological target for anti-angiogenic activity

A
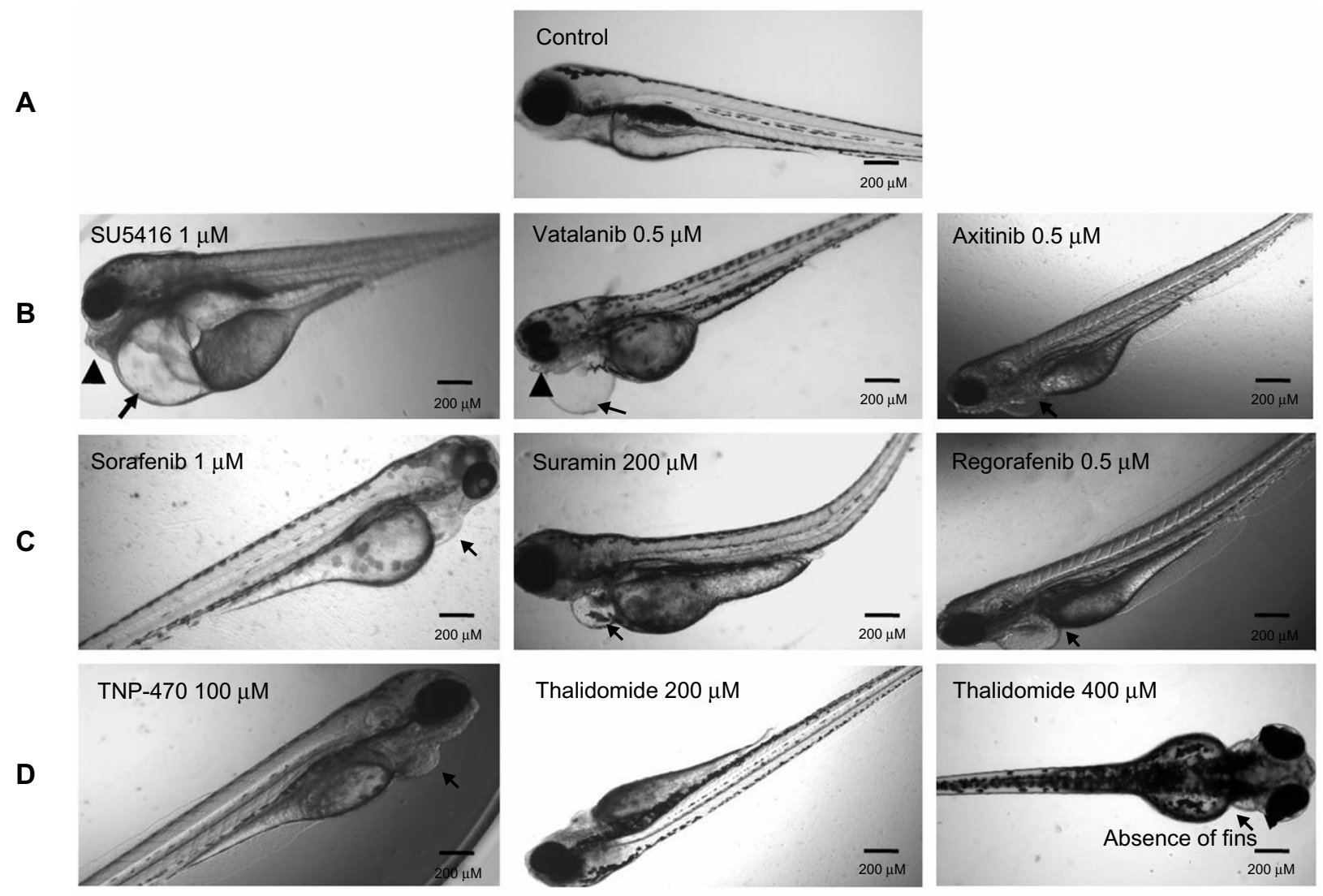

Figure 4 Gross morphological changes in the zebrafish larvae following anti-angiogenic compound exposure at 96 hours.

Notes: Each compound is evaluated at five different concentrations, with 36 embryos/concentration and compound exposure for 96 hours. (A) Control larvae: normal phenotype. (B) VEGFR inhibitors: SU54I6 treatment: jaw deformity (arrowhead), pericardial edema (arrows), and un-inflated swim bladder; vatalanib treatment: jaw deformity (arrowhead) and pericardial edema (arrows), slowed circulation; axitinib treatment: pericardial edema. (C) Multikinase inhibitors: sorafenib, regorafenib treatment: pericardial edema (arrows); suramin treatment: bent notochord, pericardial edema (arrows). (D) Inhibitors of endothelial cell proliferation: TNP-470 treatment: pericardial edema (arrows); thalidomide treatment: absence of fin development at $400 \mu \mathrm{M}$.

Abbreviation: VEGFR, vascular endothelial growth factor receptor. 
(Figure 4). Treatment with VEGFR inhibitors expressed cardiac phenotype responses and resulted in progressive pericardial edema as the gross morphological feature in all the zebrafish embryos subjected to treatment (ie, 36/36) (Figure S1). Progressive pericardial edema represented a VEGFR-inhibition phenotype associated exclusively with VEGFR inhibitors. In addition to the progressive pericardial edema, amongst the VEGFR inhibitors, treatment with SU5416 and vatalanib only was associated with multiple gross morphological abnormalities like yolk-sac edema, shortened body axis, bent notochord, jaw deformity, blood pooling, and un-inflated swim bladder (Figure 4).

Treatment with multikinase inhibitors sorafenib and regorafenib was associated with nonprogressive pericardial edema in all the embryos subjected to treatment (ie, 36/36). However, treatment with multikinase inhibitors like flavopiridol and suramin resulted in multiple sub-lethal toxicities like yolk-sac edema, nonprogressive pericardial edema, bent notochord, and jaw deformity. Widespread tissue necrosis was observed with flavopiridol treatment, and treatment with combretastatin was associated with coagulation of embryos (Figure S2).

Treatment with TNP-470 was associated with abnormal notochord morphology, while thalidomide treatment (up to permissible limits of solubility) did not result in gross morphological changes with respect to notochord development and development of cardiac edema. The only changes observed in thalidomide-treated zebrafish embryos were reduction of otolith size and altered fin development at concentrations $>300 \mu \mathrm{M}$ (Figure S3).

To match the experimental set-up for an angiogenesis assay for generation of an unbiased therapeutic window, toxicity studies were initiated at $24 \mathrm{hpf}$ for the anti-angiogenic agents evaluated. Additional toxicity studies monitoring time dependency could further elucidate toxicities associated with the anti-angiogenic drugs evaluated.

\section{Therapeutic window}

The therapeutic window represents the range of therapeutic concentrations within the LOEC of anti-angiogenic agents. The therapeutic window was analyzed to evaluate the zebrafish efficacy-toxicity profile, with the current clinical status of anti-angiogenic agents wherein the antiangiogenic agents were dropped from clinical trials due to lack of a therapeutic window or unacceptable organ-specific toxicities.

For VEGFR inhibitors, the anti-angiogenic concentrations were almost half the respective LOEC concentrations, suggestive of a wider therapeutic window for these drugs except for vatalanib and SU5416 (Figure 5). For SU5416 and vatalanib the effective therapeutic concentrations were equal to the LOEC concentrations, suggesting simultaneous occurrence

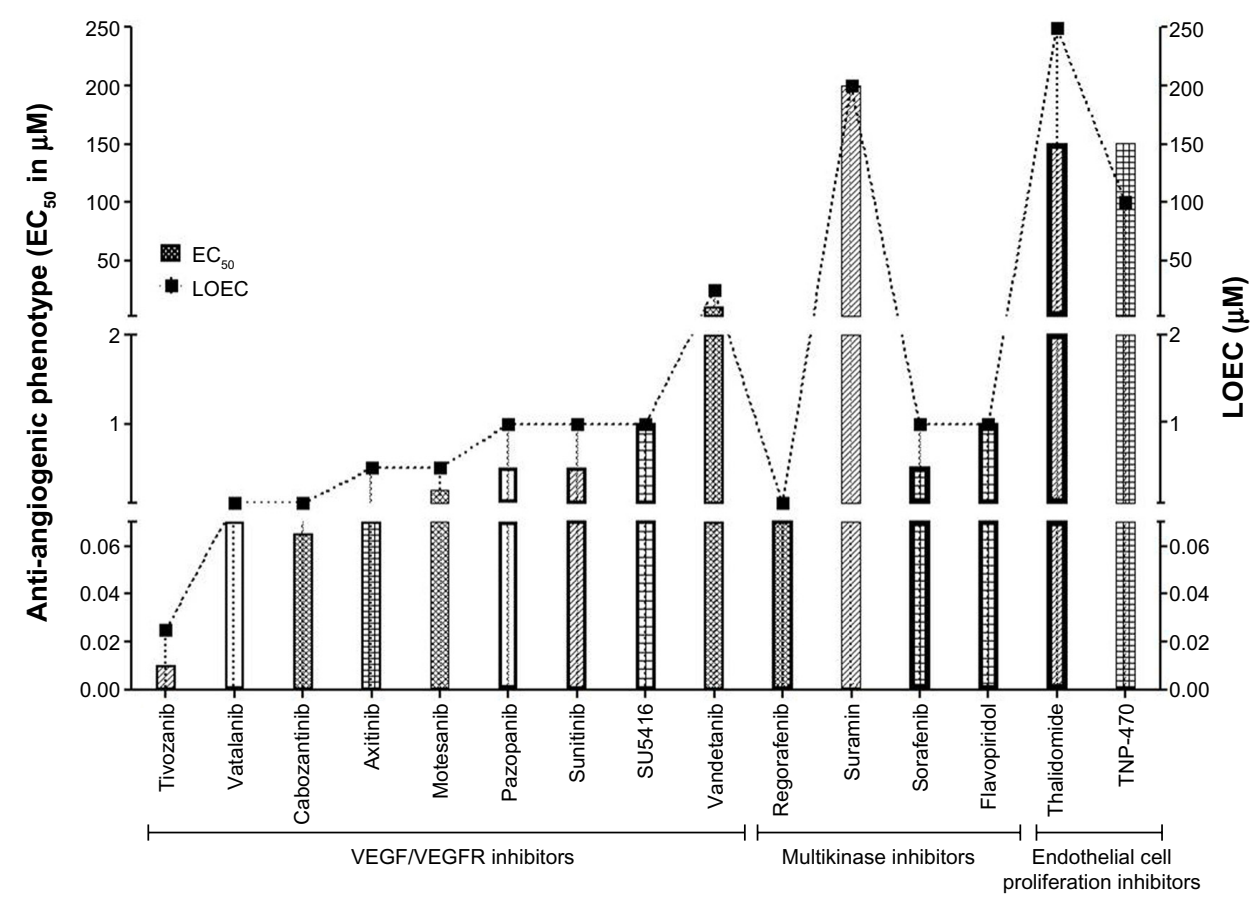

Figure 5 Therapeutic windows as exhibited by the anti-angiogenic compounds.

Abbreviations: VEGF, vascular endothelial growth factor; VEGFR, vascular endothelial growth factor receptor; LOEC, lowest observed effect concentration; EC ${ }_{50}$, half maximal effective concentration. 
of the toxic effects with the desired therapeutic effects. Thus amongst the VEGFR inhibitors the therapeutic window was absent only for SU5416 and vatalanib, suggesting presence of therapeutic windows for all other VEGFR inhibitors evaluated (Figure 5).

Amongst the multikinase inhibitors, wider therapeutic windows were demonstrated by sorafenib and regorafenib only. Treatment with flavopiridol or suramin was associated with absence of therapeutic window and multiple gross morphological changes. The therapeutic window was demonstrated by the endothelial cell proliferation inhibitor thalidomide, but was absent in zebrafish treated with TNP-470. TNP-470 exhibited anti-angiogenic efficacy at concentrations higher than the sub-lethal toxic concentrations.

The zebrafish efficacy-toxicity profile suggests that SU5416, flavopiridol, suramin, vatalanib, and TNP-470 could be associated with serious adverse effects in clinics in comparison with sunitinib, sorafenib, motesanib, pazopanib, vandetanib, tivozanib, thalidomide, axitinib, cabozantinib, and regorafenib. The therapeutic window could not be computed for combretastatin, bevacizumab, and erlotinib and hence these agents were not included in the analysis (Figure 5).

\section{Discussion}

In this study, we evaluate the suitability of zebrafish as a translational efficacy-toxicity model in anti-angiogenic drug screening. This is, to the best of our knowledge, the first exhaustive study to report stratification of anti-angiogenic agents on mechanism-based assortment of anti-angiogenic activity and toxicity.

The results of the present study demonstrate that targeted anti-angiogenic agents like VEGFR inhibitors are the most potent inhibitors of angiogenesis in the zebrafish model, with tivozanib resulting in complete obliteration of SIVs at $5 \mathrm{nM}$, followed by axitinib $\left(\mathrm{EC}_{50}=100 \mathrm{nM}\right)$. Except for vandetanib $\left(\mathrm{EC}_{50}=10 \mu \mathrm{M}\right)$, all the VEGFR inhibitors exhibited $\mathrm{EC}_{50} \leq 500 \mathrm{nM}$. The differential anti-angiogenic potency exhibited by the VEGFR inhibitors in the zebrafish assay correlates well with their reported differential VEGFRinhibition potency in biochemical assays, where tivozanib $\left(\text { VEGFR IC }_{50} \sim 0.16-0.24 \mathrm{nM}\right)^{18,19}$ and axitinib ${ }^{20}$ are the most potent VEGFR inhibitors while vandetanib exhibits the lowest VEGFR inhibition $\left(\mathrm{IC}_{50} \sim 40-108 \mathrm{nM}\right) .{ }^{21}$

Treatment with VEGFR inhibitors resulted in complete inhibition of zebrafish SIV angiogenesis, emphasizing their superior anti-angiogenic potential. This differentiation obtained in the zebrafish assay is in agreement with the in vitro endothelial cell migration and tube formation assays with human primary endothelial cells, wherein the VEGFR inhibitors exhibited anti-angiogenic potential at nanomolar concentrations $^{22,23}$ while other anti-angiogenic agents like multikinase inhibitors suramin and flavopiridol, and endothelial cell proliferation inhibitors like thalidomide, were effective at micromolar concentrations. ${ }^{24,25}$

The potent in vitro angiogenesis inhibition by antiangiogenic agents like TNP-470 and combretastatin is due to their nonselective cytotoxicity towards the endothelial cells rather than true inhibition of VEGF-driven angiogenesis. TNP-470 exhibits weaker anti-angiogenic potency in in vivo angiogenesis models in comparison with other antiangiogenic agents included in the study. ${ }^{26}$

The inhibitory effects of anti-angiogenic compounds in the zebrafish assay are also in conformity with in vivo angiogenesis assays like the CAM assay, the rat corneal micropocket assay, as well as the xenograft angiogenesis assay. ${ }^{20,23,27-31}$ VEGFR inhibitors exhibit potent inhibition of angiogenesis in the CAM assay at $1-2 \mu \mathrm{M}$, while other anti-angiogenic agents like suramin and TNP-470 inhibit angiogenesis at 7-fold and 100-fold higher concentration in the CAM assay at $7 \mu \mathrm{M}^{32}$ and $100-125 \mu \mathrm{M}$, respectively. ${ }^{26}$ Thalidomide does not exhibit anti-angiogenic effects in the CAM assay due to absence of metabolic machinery required for generation of active thalidomide metabolite responsible for its anti-angiogenic activity. ${ }^{33}$

The only exceptions to the mechanism-based anti-angiogenic activity in the zebrafish assay were the anti-angiogenic effects exhibited by the multikinase inhibitors sorafenib and regorafenib. Treatment with sorafenib and regorafenib resulted in complete inhibition of SIV angiogenesis, an experimental feature not achieved by any non-VEGFR angiogenesis inhibitor. This could be attributed to differences in mechanisms of angiogenesis inhibition associated with sorafenib, regorafenib, and other multikinase inhibitors and endothelial cell proliferation inhibitors. Akin to VEGFR inhibitors, sorafenib and regorafenib are associated with potent VEGFR inhibition in biochemical assays (sorafenib: $\mathrm{IC}_{50} \sim 26-100 \mathrm{nM}$; regorafenib: $\left.\mathrm{IC}_{50} \sim 13-46 \mathrm{nM}\right)^{29,34}$ and exhibit significant angiogenesis inhibition in the in vivo CAM assay at $1-3 \mu \mathrm{M} .{ }^{35,36}$

Other multikinase inhibitors do not exhibit potent VEGFR inhibition as a mechanism of angiogenesis inhibition. Suramin inhibits angiogenesis driven by basic fibroblast growth factor-induced endothelial cell proliferation, ${ }^{25}$ while flavopiridol inhibits hypoxia-inducible factor $1 \alpha$-stimulated VEGF secretion, resulting in inhibition of angiogenesis. ${ }^{37}$ 
This study demonstrated that the mechanism-driven differential anti-angiogenic potency can be elucidated by the zebrafish SIV angiogenesis inhibition assay and correlates well with the biochemical assays, in vitro and in vivo angiogenesis assays like the CAM assay and the matrigel plug assay. ${ }^{18-20,23,25,27,29-31}$

Angiogenesis inhibition was not demonstrated by bevacizumab, erlotinib, or combretastatin. Absence of anti-angiogenic efficacy with bevacizumab ${ }^{38}$ could be attributed to the lack of binding of bevacizumab (humanized monoclonal antibody) to the zebrafish ortholog ${ }^{39,40}$ or could be due to the inability of monoclonal antibodies to permeate sufficiently to have an effect on the SIVs. Further evaluation of the anti-angiogenic efficacy of other vascular-disrupting biological therapies like aflibercept, a fusion protein and inhibitor for VEGF, and ramicirumab, an antibody to VEGFR2, could offer insight on the suitability of the zebrafish model to predict the probable outcome of biological agents and warrant investigation. Evaluation of the anti-angiogenic potential of erlotinib was restricted as it precipitated at concentrations $>1 \mu \mathrm{M}$. Combretastatin did not inhibit angiogenesis in the zebrafish assay and is also known to have no role in affecting tumor angiogenesis in clinical trials..$^{41,42}$

This study demonstrates that unlike the qualitative in vitro assays, we can quantitatively demonstrate efficacy-based stratification of angiogenesis inhibitors. Angiogenesis inhibition was exhibited by 15 of the 18 anti-angiogenic agents known to cause angiogenesis inhibition in the clinical setting. The obtained results correlate well with their reported differential angiogenesis-inhibition potency in the preclinical angiogenesis assays and the clinical outcomes associated with the use of the anti-angiogenic agents. Tivozanib, which was the most potent anti-angiogenic agent in the zebrafish assay in comparison with other anti-angiogenic agents like sorafenib and sunitinib, has indeed demonstrated improved clinical performance in patients with renal cell carcinoma by improving progression-free survival by $\sim 12$ months ${ }^{43}$ in comparison with sorafenib ${ }^{44}$ and sunitinib ${ }^{43}$ and other VEGFR inhibitors. ${ }^{45}$ The results of this study suggest a high degree of sensitivity for screening anti-angiogenic agents in the zebrafish.

Given the heterogeneity of tissues affected by new blood vessel formation as well as the molecular and cellular differences in the angiogenic reactions, it has been a challenge to identify an ideal angiogenesis assay encompassing the multitude of features associated with angiogenesis. ${ }^{46}$ The in vivo angiogenesis assays like the CAM assay and the corneal micropocket assay are associated with several limitations to allow extensive integration in anti-angiogenic drug screening and translational drug discovery. The CAM assay, though informative and timeintensive, is associated with artifactual changes in vascular density associated with embryonic development, necessitating involvement of two or more independent observers to prevent bias in interpretation. The compound application in the CAM assay is often complicated by the use of carriers like Elvax $^{\circledR}$ (DuPont Co, Wilmington, Delware, USA) or Hydron ${ }^{\circledR}$ (Hydron Labs Inc., New Brunwick, New Jersey, USA), which can independently affect angiogenesis by controlling the rate and extent of drug release. Systemic injection of the drugs requires considerable skilled personnel engagement and is often associated with trauma-induced inflammation resulting in altered pattern of angiogenesis. Drugs that require metabolic activation cannot be evaluated in the CAM assay and result in false negatives. ${ }^{47}$

The corneal micropocket assay overcomes some of the shortcomings of the CAM assay by providing presence of metabolic machinery to study effect of active metabolites and offering easier access to injection sites for systemic administration, but encounters ethical problems with the utilization of major sensory organs and demanding as well as timeconsuming surgical techniques, excluding its employment in early and translational anti-angiogenic drug discovery for screening agents. Moreover, like the CAM assay the corneal micropocket assay is also susceptible to atypical angiogenesis and nonspecific inflammatory response. The close exposure of the newly developing blood vessels to higher oxygen concentration at the tissue-air interface in the CAM assay and the corneal micropocket assay can independently affect the new vessel formation process. ${ }^{48}$

In the existing scenario, in vivo angiogenesis assays with translational potential that are reliable, repeatable, quantitative, and amenable to compound screening are becoming an imperative in the field of anti-angiogenic drug discovery. In this context, the zebrafish assay is technically simple and truly quantitative, offering a large number of animals for analysis. The zebrafish have a matured and metabolically active liver at $72 \mathrm{hpf}$ and can be coupled with exogenous mammalian metabolic activation system (mDarT) to detect anti-angiogenic agents with active metabolites. ${ }^{49}$ Since the compounds are bath-applied to the zebrafish, trauma-associated inflammatory events are precluded in the zebrafish assay.

The findings of this current study and their agreement with the clinical and preclinical outcomes of the evaluated anti-angiogenic agents suggest utilization of zebrafish as 
an alternative approach to the existing preclinical methods for anti-angiogenic drug discovery. However, compound solubility in the test medium requires attention as this study also demonstrates that compound solubility, cross-species antibody binding, and permeability remain some of the drawbacks of the zebrafish assay in translational drug discovery. Caution is also warranted as the zebrafish model is a nonmammalian embryonic model and differences in the expression pattern of molecular markers between the zebrafish and humans is known. ${ }^{50}$ Effect of developmental physiology on angiogenesis, such as influence of embryonic stem cells, requires consideration during evaluation of results. Unlike the CAM assay or the matrigel plug assay, evaluation of basic fibroblast growth factor-induced and VEGF-induced angiogenesis in the zebrafish may remain a challenge.

In addition to evaluation of anti-angiogenic efficacy, the goal of the study was to simultaneously evaluate toxicity to predict the therapeutic window by performing efficacy and toxicity in conjunction. To this end, the toxicity profile of the compounds typifying mechanism-based class effects was generated. VEGFR inhibitors exhibited progressive pericardial edema as a gross morphological change, suggestive of cardiotoxic potential for VEGFR inhibitors. This toxicology finding in the zebrafish assay concurs with clinical findings associated with the use of VEGFR inhibitors. Treatment with sunitinib was associated with congestive cardiac failure in $11 \%$ of patients and decrease in left ventricular ejection fraction in $28 \%$ of patients enrolled in clinical trial. ${ }^{51}$ Decrease in left ventricular cardiac function and hypertension are also observed in patients treated with VEGFR inhibitors like pazopanib and the multikinase inhibitor with potent VEGFR inhibition sorafenib. ${ }^{23,24}$ Progressive pericardial edema could be the phenotypic expression of cardiovascular pathology associated with use of VEGF-targeted therapeutics in the zebrafish and could be used to generate target-based compound clusters (VEGF axis inhibitors versus non-VEGF inhibitors). Progressive pericardial edema is the characteristic feature observed in VEGF-A knockdown zebrafish embryos. ${ }^{25}$ Bevacizumab, antibody to VEGF, did not elicit dose-dependent toxicity or a consistent pathological phenotype in the zebrafish assay. The aforementioned toxicity results also compare with the lack of anti-angiogenic efficacy associated with bevacizumab in the zebrafish assay. This phenomenon is attributed to lack of permeability or cross-species antibody binding, once again demonstrating that permeability or differences in cross-species binding domains may remain limitations of the zebrafish assays. Amongst the VEGFR inhibitors evaluated multiple morphological changes were observed in the zebrafish embryos treated with SU5416 and vatalanib, suggesting inhibition of a wider range of biological pathways other than the specific VEGFR pathway, which could be attributed to chemical structure-associated disparity in the biological activity.

Presence of multiple pathological changes like jaw deformity, pericardial edema, notochord deformity, and patchy necrosis were also observed with multikinase inhibitors flavopiridol and suramin and are suggestive of potential for inhibition of multiple biological targets resulting in multiple off-target effects. Akin to the zebrafish results, flavopiridol administration in clinical trials is associated with several toxicities, including tumor lysis syndrome and febrile neutropenia ${ }^{52}$ while suramin is associated with neutropenia, neurological dysfunction, and development of renal insufficiency. ${ }^{53}$ Treatment with combretastatin was associated with coagulation of zebrafish embryos, emphasizing the nonselective endothelial cell toxicity of this agent. Thalidomide treatment exhibited reduction of otolith size and altered fin development at concentrations $>300 \mu \mathrm{M}$, in agreement with reported literature. ${ }^{54}$

The zebrafish toxicity studies demonstrated the cardiotoxic potential of VEGFR inhibitors and multiple systemic toxicities associated with other classes of anti-angiogenic agents evaluated. Thus, in a blinded scenario, where it is difficult to predict outcome in a clinical setting, the zebrafish efficacy-toxicity model can be a no-go tool for compound selection, simultaneously establishing the strength and derangement associated with the biological target under consideration.

To further evaluate the suitability of zebrafish efficacytoxicity model in translational anti-angiogenic drug discovery, we evaluate the potential of this model to predict therapeutic windows. The therapeutic window was evaluated with LOEC concentrations. As anti-angiogenic agents require chronic administration, necessitating a higher margin of safety, LOEC concentrations observed with chronic dosing were considered for predicting the therapeutic window instead of the $\mathrm{LC}_{50}$ concentrations associated with mortality. The performance of the therapeutic window as a predictive assay parameter was measured by sensitivity (true positives) and specificity (true negative) with respect to the reported clinical trial outcomes of the individual compounds.

The zebrafish efficacy-toxicity model demonstrated therapeutic windows for sorafenib, sunitinib, motesanib, pazopanib, vandetanib, thalidomide, and tivozanib. These results are in accordance with the clinical trial outcomes for sorafenib, sunitinib, pazopanib, thalidomide, and vandetanib 
(ie, 7/7 match). ${ }^{26-32}$ Therapeutic windows were observed for the aforementioned anti-angiogenic agents in preclinical xenograft and xenograft angiogenesis experiments and also for anti-angiogenic agents tivozanib and motesanib, which are under clinical trial investigation. ${ }^{18,23,29,55}$ Sorafenib, sunitinib, pazopanib, vandetanib, and thalidomide have become approved drugs with acceptable therapeutic windows, while tivozanib and motesanib in Phase III clinical trials have exhibited improvement in progression-free survival and overall survival. ${ }^{56,57}$

Absence of a therapeutic window was proposed for SU5416, vatalanib, flavopiridol, suramin, and TNP-470, which is in accordance with clinical trial outcomes for these aforementioned compounds (ie, 5/5 match). Development of SU5416, vatalanib, flavopiridol, suramin, and TNP-470 has been suspended due to dose-limiting toxicities. The lack of a therapeutic window for TNP-470 was also demonstrated in early discovery in the mouse dorsal air sac model for angiogenesis wherein the effectiveness of TNP-470 was limited by severe weight loss. ${ }^{58}$ For SU5416, the preclinical efficacy studies demonstrated severe hemorrhagic necrosis of tumor tissue at antitumor concentrations; however, there was lack of further evidence of toxicity in the preclinical studies. ${ }^{27,59}$ In the clinical setting, SU5416 offered no benefits to the patients at the targeted dose, and dose-escalation studies to attain higher desirable concentrations were associated with undesirable adverse effects. ${ }^{60}$ Combretastatin also elicited a similar clinical response, wherein tumor necrosis due to damage to tumor blood vessels was observed at doses that were too toxic for patients to tolerate. ${ }^{61}$ The lack of a therapeutic window in non-VEGFR inhibitors like suramin, flavopiridol, combretastatin, and SU5416 has been attributed to inhibition of a wide range of targets other than the VEGFR, which ultimately disrupts several signaling pathways and causes off-target toxicities.

Thus, the results of this study suggest a high degree of sensitivity and specificity for therapeutic windows in the zebrafish as an efficacy-toxicity model and are in agreement with the reported outcomes of clinical trials and preclinical studies to be implemented in translational anti-angiogenic drug discovery. Thus, the zebrafish efficacy-toxicity model can be used to predict the probable outcome of anti-angiogenic agents.

In summary, this report for the first time demonstrates the ability of zebrafish embryos to stratify anti-angiogenic agents based on their mechanism of action, typify a class effect in zebrafish embryo toxicity assays, and delineate chemical structure-based driven differentiation of biological activity for compounds with a similar mechanism of action. Therapeutic windows as a parameter can provide a sensitive and specific read-out for streamlining compound selection in early anti-angiogenic drug discovery. In addition to the data presented here, the zebrafish embryos are amenable to molecular mechanistic studies and can be further explored to enhance the understanding of molecular interactions between anti-angiogenic agents and different biological pathways affected.

Zebrafish can be utilized to further evaluate newer angiogenic targets and their role in zebrafish angiogenesis regulation. The existing assay evaluates only the process of natural angiogenesis and vasculogenesis. To further enhance its potential as a powerful screening tool for anti-angiogenic drug discovery, it should be combined with a zebrafish tumor xenograft angiogenesis screen, which also models the phenomenon of neoangiogenesis. ${ }^{62}$

The zebrafish are amenable to cancer cell grafts and exhibit differentiation between highly angiogenic and poorly angiogenic tumors. ${ }^{63}$ Due to their ability to bear xenografts and exhibit clinically relevant differential angiogenic behavior, the zebrafish can be subjected to pulse or metronomic regimen of anti-angiogenic agents to study acquired drug resistance to anti-angiogenic therapies and investigate the underlying molecular mechanisms for the escape phenomenon. This extension of the zebrafish angiogenesis assay has multiple advantages over the existing murine tumor angiogenesis models with respect to compound requirement, duration of study, and ability to screen compounds.

Given the ability to generate a therapeutic window, quantitative angiogenic read-out, and a pathological phenotype in early drug discovery, the zebrafish efficacy-toxicity assay could offer a quick translational tool to facilitate antiangiogenic drug discovery.

\section{Author contributions}

All authors contributed toward data analysis, drafting and revising the paper and agree to be accountable for all aspects of the work.

\section{Disclosure}

The authors report no conflict of interest in this work.

\section{References}

1. Folkman J. Angiogenesis in cancer, vascular, rheumatoid and other disease. Nat Med. 1995;1(1):27-31.

2. Verheul HM, Pinedo HM. Inhibition of angiogenesis in cancer patients. Expert Opin Emerg Drugs. 2005;10(2):403-412. 
3. Pasquier E, Kavallaris M, Andre N. Metronomic chemotherapy: new rationale for new directions. Nat Rev Clin Oncol. 2010;7(8): $455-465$.

4. Faivre S, Delbaldo C, Vera K, et al. Safety, pharmacokinetic, and antitumor activity of SU11248, a novel oral multitarget tyrosine kinase inhibitor, in patients with cancer. J Clin Oncol. 2006;24(1):25-35.

5. Verheul HM, Pinedo HM. Possible molecular mechanisms involved in the toxicity of angiogenesis inhibition. Nat Rev Cancer. 2007;7(6): 475-485.

6. Elice F, Rodeghiero F, Falanga A, Rickles FR. Thrombosis associated with angiogenesis inhibitors. Best Pract Res Clin Haematol. 2009;22(1): 115-128.

7. Rajotte D, Arap W, Hagedorn M, Koivunen E, Pasqualini R, Ruoslahti E. Molecular heterogeneity of the vascular endothelium revealed by in vivo phage display. J Clin Invest. 1998;102(2):430-437.

8. Staton CA, Stribbling SM, Tazzyman S, Hughes R, Brown NJ, Lewis CE. Current methods for assaying angiogenesis in vitro and in vivo. Int $J$ Exp Pathol. 2004;85(5):233-248.

9. Serbedzija GN, Flynn E, Willett CE. Zebrafish angiogenesis: a new model for drug screening. Angiogenesis. 1999;3(4):353-359.

10. Lyons MS, Bell B, Stainier D, Peters KG. Isolation of the zebrafish homologues for the tie-1 and tie-2 endothelium-specific receptor tyrosine kinases. Dev Dyn. 1998;212(1):133-140.

11. Liang D, Chang JR, Chin AJ, et al. The role of vascular endothelial growth factor (VEGF) in vasculogenesis, angiogenesis, and hematopoiesis in zebrafish development. Mech Dev. 2001;108(1-2):29-43.

12. Chan J, Bayliss PE, Wood JM, Roberts TM. Dissection of angiogenic signaling in zebrafish using a chemical genetic approach. Cancer Cell. 2002;1(3):257-267.

13. Cross LM, Cook MA, Lin S, Chen JN, Rubinstein AL. Rapid analysis of angiogenesis drugs in a live fluorescent zebrafish assay. Arterioscler Thromb Vasc Biol. 2003;23(5):911-912.

14. Kimmel CB, Ballard WW, Kimmel SR, Ullmann B, Schilling TF. Stages of embryonic development of the zebrafish. Dev Dyn. 1995;203(3):253-310.

15. Nicoli S, Presta M. The zebrafish/tumor xenograft angiogenesis assay. Nat Protoc. 2007;2(11):2918-2923.

16. Nagel R. DarT: The embryo test with the Zebrafish Danio rerio a general model in ecotoxicology and toxocology. ALTEX. 2002; 19(Supp 1):38-48.

17. Gridelli C, Bareschino MA, Schettino C, Rossi A, Maione P, Ciardiello F. Erlotinib in non-small cell lung cancer treatment: current status and future development. Oncologist. 2007;12(7):840-849.

18. Eskens FA, de Jonge MJ, Bhargava $P$, et al. Biologic and clinical activity of tivozanib (AV-951, KRN-951), a selective inhibitor of VEGF receptor-1, -2, and -3 tyrosine kinases, in a 4-week-on, 2-week-off schedule in patients with advanced solid tumors. Clin Cancer Res. 2011;17(22):7156-7163.

19. Pal SK, Bergerot PG, Figlin RA. Tivozanib: current status and future directions in the treatment of solid tumors. Expert Opin Investig Drugs. 2012;21(12):1851-1859.

20. Hu-Lowe DD, Zou HY, Grazzini ML, et al. Nonclinical antiangiogenesis and antitumor activities of axitinib (AG-013736), an oral, potent, and selective inhibitor of vascular endothelial growth factor receptor tyrosine kinases 1, 2, 3. Clin Cancer Res. 2008;14(22):7272-7283.

21. Morabito A, Piccirillo MC, Falasconi F, et al. Vandetanib (ZD6474), a dual inhibitor of vascular endothelial growth factor receptor (VEGFR) and epidermal growth factor receptor (EGFR) tyrosine kinases: current status and future directions. Oncologist. 2009;14(4): 378-390.

22. Kumar R, Knick VB, Rudolph SK, et al. Pharmacokineticpharmacodynamic correlation from mouse to human with pazopanib, a multikinase angiogenesis inhibitor with potent antitumor and antiangiogenic activity. Mol Cancer Ther. 2007;6(7):2012-2021.

23. Polverino A, Coxon A, Starnes C, et al. AMG 706, an oral, multikinase inhibitor that selectively targets vascular endothelial growth factor, platelet-derived growth factor, and kit receptors, potently inhibits angiogenesis and induces regression in tumor xenografts. Cancer Res. 2006;66(17):8715-8721.
24. Ng SS, Gütschow M, Weiss M, et al. Antiangiogenic activity of $\mathrm{N}$-substituted and tetrafluorinated thalidomide analogues. Cancer Res. 2003;63(12):3189-3194.

25. Danesi R, Del Bianchi S, Soldani P, et al. Suramin inhibits bFGF-induced endothelial cell proliferation and angiogenesis in the chick chorioallantoic membrane. Br J Cancer. 1993;68(5):932-938.

26. Minischetti M, Vacca A, Ribatti D, et al. TNP-470 and recombinant human interferon-alpha2a inhibit angiogenesis synergistically. $\mathrm{Br} J$ Haematol. 2000;109(4):829-837.

27. Davis DW, Takamori R, Raut CP, et al. Pharmacodynamic analysis of target inhibition and endothelial cell death in tumors treated with the vascular endothelial growth factor receptor antagonists SU5416 or SU6668. Clin Cancer Res. 2005;11(2 Pt 1):678-689.

28. Kumar K, Bhargava P, Roy U. In vitro refolding of triosephosphate isomerase from L. donovani. Appl Biochem Biotechnol. 2011;164(7): 1207-1214.

29. Wilhelm SM, Dumas J, Adnane L, et al. Regorafenib (BAY 73-4506): a new oral multikinase inhibitor of angiogenic, stromal and oncogenic receptor tyrosine kinases with potent preclinical antitumor activity. Int J Cancer. 2011;129(1):245-255.

30. Kerr JS, Wexler RS, Mousa SA, et al. Novel small molecule alpha $\mathrm{V}$ integrin antagonists: comparative anti-cancer efficacy with known angiogenesis inhibitors. Anticancer Res. 1999;19(2A):959-968.

31. Brüsselbach S, Nettelbeck DM, Sedlacek HH, Müller R. Cell cycle-independent induction of apoptosis by the anti-tumor drug Flavopiridol in endothelial cells. Int J Cancer. 1998;77(1):146-152.

32. Melkonian G, Munoz N, Chung J, Tong C, Marr R, Talbot P. Capillary plexus development in the day five to day six chick chorioallantoic membrane is inhibited by cytochalasin D and suramin. J Exp Zool. 2002;292(3):241-254.

33. Bauer KS, Dixon SC, Figg WD. Inhibition of angiogenesis by thalidomide requires metabolic activation, which is species-dependent. Biochem Pharmacol. 1998;55(11):1827-1834.

34. Wilhelm SM, Carter C, Tang L, et al. BAY 43-9006 exhibits broad spectrum oral antitumor activity and targets the RAF/MEK/ERK pathway and receptor tyrosine kinases involved in tumor progression and angiogenesis. Cancer Res. 2004;64(19):7099-7109.

35. Pignochino Y, Grignani G, Cavalloni G, et al. Sorafenib blocks tumour growth, angiogenesis and metastatic potential in preclinical models of osteosarcoma through a mechanism potentially involving the inhibition of ERK1/2, MCL-1 and ezrin pathways. Mol Cancer. 2009;8:118.

36. Martinho O, Silva-Oliveira R, Miranda-Gonçalves V, et al. In vitro and in vivo analysis of RTK inhibitor efficacy and identification of its novel targets in glioblastomas. Transl Oncol. 2013;6(2):187-196.

37. Newcomb EW, Ali MA, Schnee T, et al. Flavopiridol downregulates hypoxia-mediated hypoxia-inducible factor-1alpha expression in human glioma cells by a proteasome-independent pathway: implications for in vivo therapy. Neuro Oncol. 2005;7(3):225-235.

38. Hurwitz H, Fehrenbacher L, Novotny W, et al. Bevacizumab plus irinotecan, fluorouracil, and leucovorin for metastatic colorectal cancer. N Engl J Med. 2004;350(23):2335-2342.

39. Ferrara N, Hillan KJ, Gerber HP, Novotny W. Discovery and development of bevacizumab, an anti-VEGF antibody for treating cancer. Nat Rev Drug Discov. 2004;3(5):391-400.

40. Geberth M, Foerster F, Klare P, et al. Efficacy and safety of first-line bevacizumab (BEV) combined with paclitaxel (PAC) according to age: subpopulation analysis of a large, multicenter, non-interventional study in patients (Pts) with HER2-negative metastatic breast cancer (MBC). Cancer Res. 2009;69(24 Suppl 3):6085

41. Tozer GM, Kanthou C, Baguley BC. Disrupting tumour blood vessels. Nat Rev Cancer. 2005;5(6):423-435.

42. Bilenker JH, Flaherty KT, Rosen M, et al. Phase I trial of combretastatin a-4 phosphate with carboplatin. Clin Cancer Res. 2005;11(4): 1527-1533.

43. Motzer RJ, Hutson TE, Tomczak P, et al. Overall survival and updated results for sunitinib compared with interferon alfa in patients with metastatic renal cell carcinoma. J Clin Oncol. 2009;27(22):3584-3590. 
44. Escudier B, Eisen T, Stadler WM, et al. Sorafenib for treatment of renal cell carcinoma: final efficacy and safety results of the phase III treatment approaches in renal cancer global evaluation trial. J Clin Oncol. 2009;27(20):3312-3318.

45. Bhargava P, Robinson MO. Development of second-generation VEGFR tyrosine kinase inhibitors: current status. Curr Oncol Rep. 2011;13(2):103-111.

46. Auerbach R, Auerbach W, Polakowski I. Assays for angiogenesis: a review. Pharmacol Ther. 1991;51(1):1-11.

47. Norrby K. Drug testing with angiogenesis models. Expert Opin Drug Discov. 2008;3(5):533-549.

48. Norrby K. In vivo models of angiogenesis. J Cell Mol Med. 2006;10(3):588-612.

49. Busquet F, Nagel R, von Landenberg F, Mueller SO, Huebler N, Broschard TH. Development of a new screening assay to identify proteratogenic substances using zebrafish danio rerio embryo combined with an exogenous mammalian metabolic activation system (mDarT). Toxicol Sci. 2008;104(1):177-188.

50. Mriouah J, Boura C, Thomassin M, et al. Tumor vascular responses to antivascular and antiangiogenic strategies: looking for suitable models. Trends Biotechnol. 2012;30(12):649-658.

51. Chu TF, Rupnick MA, Kerkela R, et al. Cardiotoxicity associated with tyrosine kinase inhibitor sunitinib. Lancet. 2007;370(9604): 2011-2019.

52. Senderowicz AM. Small molecule modulators of cyclindependent kinases for cancer therapy. Oncogene. 2000;19(56): 6600-6606.

53. Eisenberger MA, Sinibaldi VJ, Reyno LM, et al. Phase I and clinical evaluation of a pharmacologically guided regimen of suramin in patients with hormone-refractory prostate cancer. J Clin Oncol. 1995;13(9): 2174-2186.

54. Ito $\mathrm{T}$, Ando H, Suzuki T, et al. Identification of a primary target of thalidomide teratogenicity. Science. 2010;327(5971):1345-1350.
55. Yoshikawa D, Ojima H, Kokubu A, et al. Vandetanib (ZD6474), an inhibitor of VEGFR and EGFR signalling, as a novel moleculartargeted therapy against cholangiocarcinoma. Br J Cancer. 2009;100(8): 1257-1266.

56. Motzer RJ, Nosov D, Eisen T, et al. Tivozanib versus sorafenib as initial targeted therapy for patients with metastatic renal cell carcinoma: results from a phase III trial. J Clin Oncol. 2013;31(30):3791-3799.

57. Scagliotti GV, Vynnychenko I, Park K, et al. International, randomized, placebo-controlled, double-blind phase III study of motesanib plus carboplatin/paclitaxel in patients with advanced nonsquamous non-small-cell lung cancer: MONET1. J Clin Oncol. 2012;30(23): 2829-2836.

58. Ingber D, Fujita T, Kishimoto S, et al. Synthetic analogues of fumagillin that inhibit angiogenesis and suppress tumour growth. Nature. 1990;348(6301):555-557.

59. Fong TAT, Shawver LK, Sun L, et al. SU5416 is a potent and selective inhibitor of the vascular endothelial growth factor receptor (Flk-1/KDR) that inhibits tyrosine kinase catalysis, tumor vascularization, and growth of multiple tumor types. Cancer Res. 1999;59(1):99-106.

60. Kuenen BC, Tabernero J, Baselga J, et al. Efficacy and toxicity of the angiogenesis inhibitor SU5416 as a single agent in patients with advanced renal cell carcinoma, melanoma, and soft tissue sarcoma. Clin Cancer Res. 2003;9(5):1648-1655.

61. Rustin GJ, Shreeves G, Nathan PD, et al. A phase Ib trial of CA4P (combretastatin A-4 phosphate), carboplatin, and paclitaxel in patients with advanced cancer. Br J Cancer. 2010;102(9):1355-1360.

62. Nicoli S, Ribatti D, Cotelli F, Presta M. Mammalian tumor xenografts induce neovascularization in zebrafish embryos. Cancer Res. 2007;67(7):2927-2931.

63. Lee SL, Rouhi P, Dahl Jensen L, et al. Hypoxia-induced pathological angiogenesis mediates tumor cell dissemination, invasion, and metastasis in a zebrafish tumor model. Proc Natl Acad Sci USA. 2009;106(46): 19485-19490 


\section{Supplementary materials}
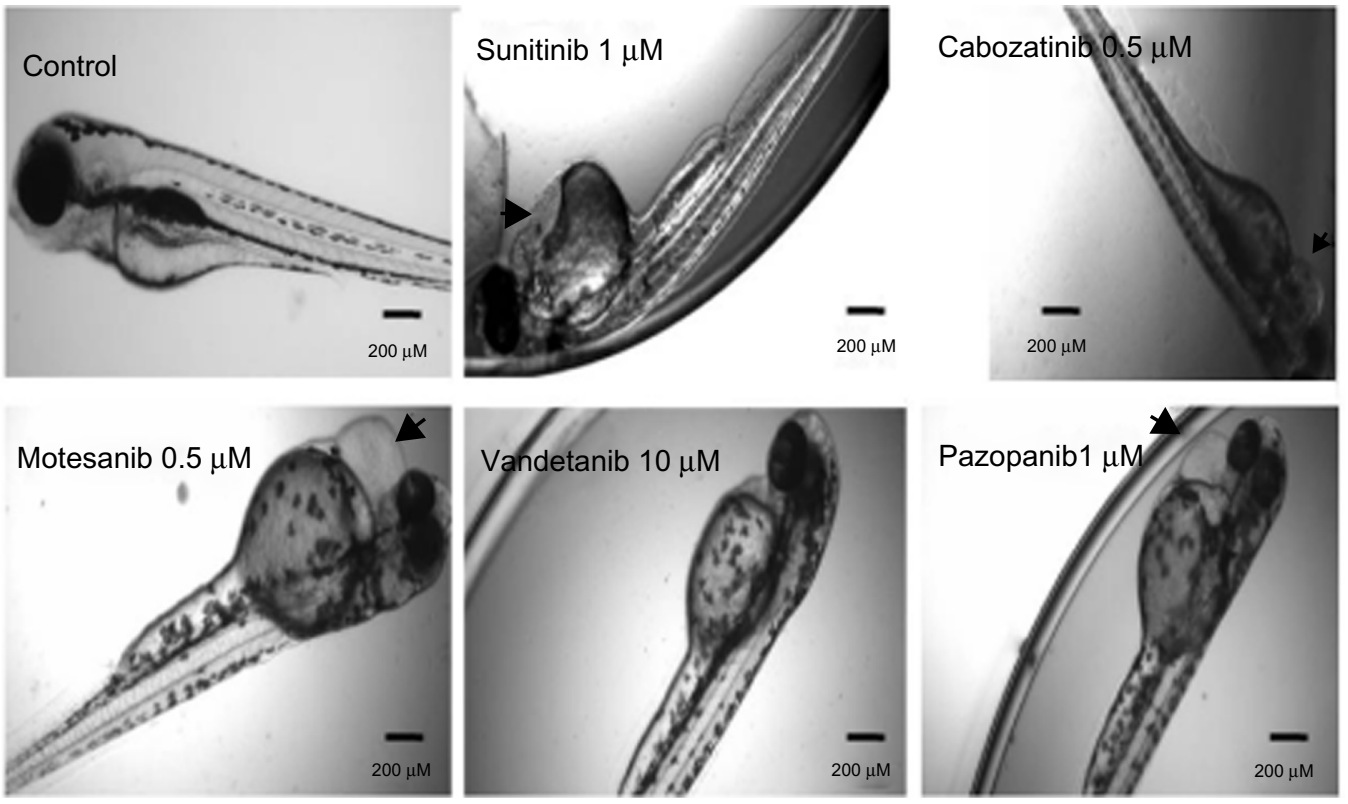

Figure SI Gross morphological changes in the zebrafish larvae following VEGFR inhibitors at 96 hours.

Notes: Treatment with VEGFR inhibitors was associated with development of pericardial edema at their LOEC concentrations. Sunitinib, cabozantinib, axitinib, motesanib, and pazopanib treatments were associated with pericardial edema at and above their LOEC concentrations (arrows). Lack of the cardiac phenotype (pericardial edema) in vandetanib treatment group at NOEC concentration.

Abbreviations: VEGFR, vascular endothelial growth factor receptor; LOEC, lowest observed effect concentration; NOEC, no observed effect concentration.
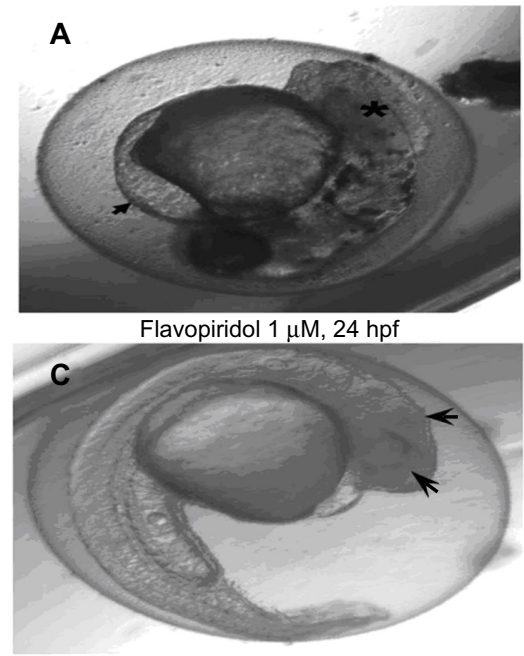

Combretastatin $5 \mathrm{nM}, 24 \mathrm{hpf}$

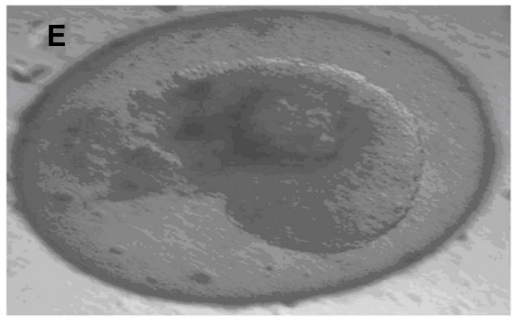

Combretastatin $10 \mathrm{nM}, 24 \mathrm{hpf}$

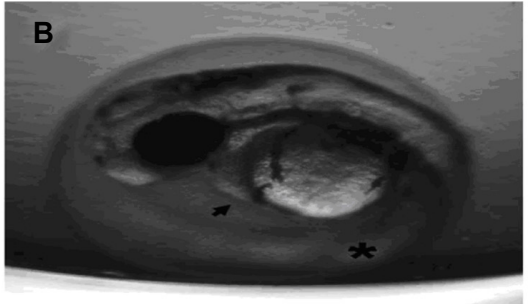

Flavopiridol $1 \mu \mathrm{M}, 48 \mathrm{hpf}$

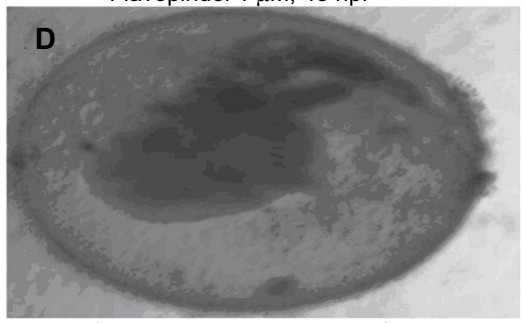

Combretastatin $5 \mathrm{nM}, 48 \mathrm{hpf}$

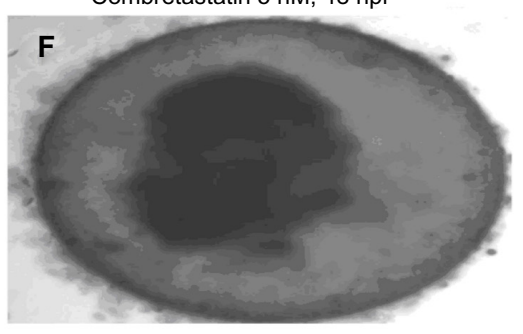

Combretastatin $10 \mathrm{nM}, 48 \mathrm{hpf}$

Figure S2 Gross morphological changes in the zebrafish larvae following non-VEGFR angiogenesis inhibitors at 96 hours post fertilization.

Notes: (A and B) Treatment with non-VEGFR inhibitors like flavopiridol was associated with development of pericardial edema (arrows) and widespread tissue necrosis (*) at LOEC concentrations. (C-F) Treatment with combretastatin was associated with dose- and time-dependent coagulation of embryos at concentrations as low as $5-10 \mathrm{nM}$ (arrows). Abbreviations: VEGFR, vascular endothelial growth factor receptor; LOEC, lowest observed effect concentration; hpf, hours post-fertilization. 


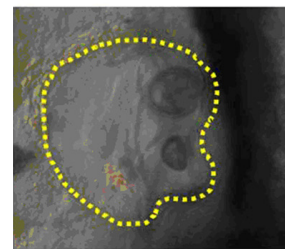

Control

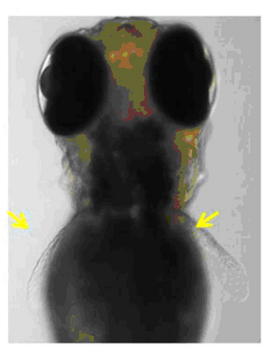

Control

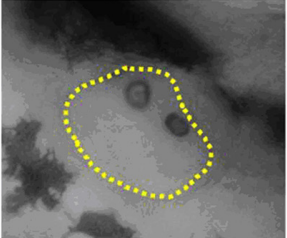

Thalidomide-treated

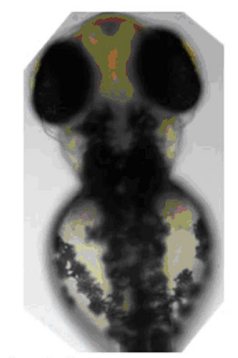

Thalidomide-treated
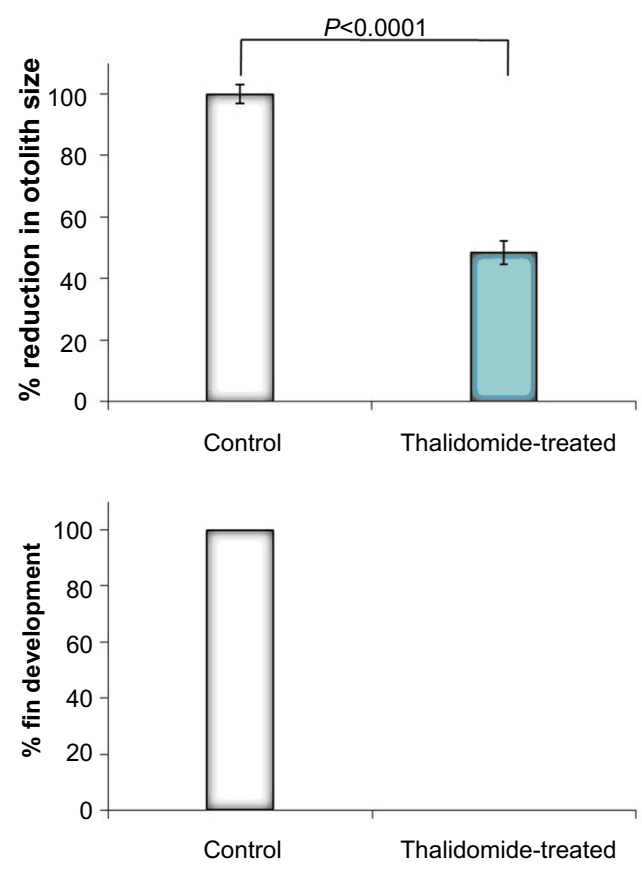

Figure S3 Gross morphological changes in the zebrafish larvae following treatment with thalidomide.

Notes: Thalidomide treatment exhibited $52 \%$ reduction in otolith size and $100 \%$ absence of fin development. Yellow arrows indicate the fin development and yellow dotted circles indicate otolith boundary.

\section{Publish your work in this journal}

Drug Design, Development and Therapy is an international, peerreviewed open-access journal that spans the spectrum of drug design and development through to clinical applications. Clinical outcomes, patient safety, and programs for the development and effective, safe, and sustained use of medicines are a feature of the journal, which has also been accepted for indexing on PubMed Central. The manuscript management system is completely online and includes a very quick and fair peer-review system, which is all easy to use. Visit http://www.dovepress.com/testimonials.php to read real quotes from published authors.

Submit your manuscript here: http://www.dovepress.com/drug-design-development-and-therapy-journal 